\title{
Brain training in progress: a review of trainability in healthy seniors
}

\author{
Jessika I. V. Buitenweg ${ }^{1 *}$, Jaap M. J. Murre ${ }^{1}$ and K. Richard Ridderinkhof ${ }^{1,2}$ \\ Department of Psychology, University of Amsterdam, Amsterdam, Netherlands \\ 2 Cognitive Science Center Amsterdam, University of Amsterdam, Amsterdam, Netherlands
}

\author{
Edited by: \\ Julia Karbach, Saarland University, \\ Germany \\ Reviewed by: \\ Michael Falkenstein, Leibniz \\ Research Centre für Working \\ Environment and Human Factors, \\ Germany \\ Céline Nadja Bürki, University of \\ Geneva, Switzerland \\ *Correspondence: \\ Jessika I. V. Buitenweg, Department \\ of Psychology, University of \\ Amsterdam, Weesperplein 4, \\ 1018 XA Amsterdam, Netherlands. \\ e-mail: j.i.v.buitenweg@uva.nl
}

\begin{abstract}
The cognitive deterioration associated with aging is accompanied by structural alterations and loss of functionality of the frontostriatal dopamine system. The question arises how such deleterious cognitive effects could be countered. Brain training, currently highly popular among young and old alike, promises that users will improve on certain neurocognitive skills, and this has indeed been confirmed in a number of studies. Based on these results, it seems reasonable to expect beneficial effects of brain training in the elderly as well. A selective review of the existing literature suggests, however, that the results are neither robust nor consistent, and that transfer and sustained effects thus far appear limited. Based on this review, we argue for a series of elements that hold potential for progress in successful types of brain training: (1) including flexibility and novelty as features of the training, (2) focusing on a number of promising, yet largely unexplored domains, such as decision-making and memory strategy training, and (3) tailoring the training adaptively to the level and progress of the individual. We also emphasize the need for covariance-based MRI methods in linking structural and functional changes in the aging brain to individual differences in neurocognitive efficiency and trainability in order to further uncover the underlying mechanisms.
\end{abstract}

\section{Keywords: brain training, aging, plasticity, adaptiveness, individual differences, executive functions, memory}

\section{INTRODUCTION}

Given the continuously growing number of elderly and their increasing longevity expectation, there is a pressing need to prolong independent functioning and to sustain quality of life by delaying the effects of cognitive decline. Human aging is typically associated with a deterioration of cognitive functioning, which is seen in multiple domains, including memory, decisionmaking, and cognitive control (Fisk and Sharp, 2004; Luo and Craik, 2008; Brown and Ridderinkhof, 2009). Decline is associated with shrinkage of prefrontal cortex, hippocampus, and basal ganglia (Raz et al., 2005) and alterations in their structural connectivity (O'sullivan et al., 2001; Madden et al., 2009) along with a decrease in synthesis and binding of dopamine, serotonin and acetylcholine (Wang et al., 1995, 1998; Volkow et al., 1998; Bäckman et al., 2006; Schliebs and Arendt, 2010). Together, these structural changes cause neuromodulator levels to drop, affecting important functional pathways, principally in striatal and frontostriatal areas (Bäckman et al., 2006).

A number of interventions have been suggested to slow down this decline. Offering a motivational incentive has been demonstrated to have beneficial effects on cognitive performance (Harsay et al., 2010), and individual differences in this benefit were related to several frontostriatal white matter pathways (Harsay et al., 2011). Aerobic exercise has also been shown to aid in maintaining cognitive health by reducing age-related loss and adding to volume of grey and white matter in frontal and temporal cortices (Colcombe et al., 2003, 2006). Recent DTI studies suggest a relation between exercise and increased fractional anisotropy (FA) in white matter tracts (Marks et al., 2007; Voss et al., 2010). Another set of interventions concerns mental stimulation, collectively known as brain training: activities intended to challenge cognitive abilities and induce learning. Unfortunately, the many different brain training studies employ a range of varying methods and definitions, participants are not consistently subjected to tests of transfer and long-term retention, and evidence pointing to the trainings' effectivity is inconsistent. These limitations notwithstanding, brain training is practiced by elderly on a large scale.

An important concept in the realm of cognitive training is that of transfer, the degree to which the learned skill is displayed in a different context, with near and far transfer referring to generalization of training effects to domains proximal to or more distant from the trained skill, respectively. Recent reviews of the current brain training literature on this topic conclude that training programs generally fail to display fundamental transfer, with the exception of process-based cognitive control tasks (Lustig et al., 2009; Noack et al., 2009; Papp et al., 2009). They comment on the limited methodology and arbitrary assignment of transfer tasks as either near or far, which make it difficult to draw conclusions on transferability. Furthermore, many studies do not make use of active controls, thus limiting the generalizability of results. Noack et al. (2009) also note that, given the fact that training programs mostly consist of no more than a few sessions of training, the transfer found in these cases is unlikely to be mediated by neural plasticity. In working-memory training, transfer effects are also seen to be small or nonexistent (Dahlin et al., 2009), although 
long-term sustained gains are reported at least for the task trained. Concerning memory, Hertzog et al. (2008) proposed that interventions should engage multiple mechanisms closely related to executive control- and other functions used by elderly on various settings in daily life.

Although a good number of articles have been written reviewing some important domains in brain training literature, we feel the need to add to the current literature by drawing attention to a number of largely unexplored perspectives, in addition to emphasizing a few promising components that may make up an effective intervention. Given the current state of brain training research in elderly, the negative overall outlook notwithstanding, a number of aspects may potentially contribute to success of future studies, which motivates our discussion of these issues in the current article. First, much attention has been paid to interventions involving cognitive control, some of which (in particular those involving cognitive flexibility) seem very promising. A systematic analysis could therefore be useful in generating an overview of the types of tasks that result in meaningful transfer and long-term retention. Second, several avenues that might well prove to be effective have yet been largely ignored in brain training research. These include decision-making and -learning, which are affected by old age and could potentially benefit from training; novelty, which prepares the neuronal system for learning and could enhance ensuing synaptic plasticity; and memory strategy training, which could transcend the memory domain and lead to far transfer. Finally, and importantly, we believe future studies can profit from a stronger emphasis on inter-individual differences in trainability. The current literature largely fails to take such individual differences and their underlying determinants into consideration. Given the massive individual differences in performance and the rate of neurocognitive decline among the elderly population, future studies thus may benefit from incorporating individual fine-tuning and adaptation into the training programs, in particular from focusing on who does and who does not benefit from a given training program, and from using neuroimaging to connect inter-individual differences in performance to perceivable differences in brain structure as well as functional connectivity and/or activation.

We will first review the current evidence on training of executive functions, arguing that persistent training of cognitive control functions can, under certain conditions, enhance performance and lead to near and far transfer. We then focus on some additional perspectives which have not yet or only modestly been implemented as an intervention, but seem to hold promise in enhancing functioning. Finally, we address the importance of recognizing the inter-individual differences in brain and behavior between elderly and its impact on cognitive training possibilities.

\section{TRAINING EXECUTIVE FUNCTIONS}

Executive functioning concerns the regulation and control of goal-directed actions. Due to the large functional dependence on prefrontal cortex and basal ganglia (Ridderinkhof et al., 2004, 2011), functions of executive control are especially prone to decline in old age (Treitz et al., 2007). It is thus reasonable to assume that training of executive functions can benefit elderly in daily life performance. This might pertain especially to tasks engaging cognitive flexibility (Buchler et al., 2008; Karbach and Kray, 2009), in other words, tasks that strengthen the general ability to adapt one's responses to the demand of the current situation and stimulate creative, novel thought. For this reason we focus strongly on executive functioning training, notably those domains that hold greatest promise in inducing flexibility. The executive functions are often divided, according to a widely adopted classification model based on latent-factor analysis by Miyake and colleagues (2000), into three separate domains of functioning: shifting, updating, and inhibition, which we will follow here.

\section{SHIFTING}

Shifting involves the flexibility to switch one's attention and one's actions between relevant tasks or subtasks, thus also dealing with interference. This is often symbolized by task switching and by multitasking. In task switching a switch is made between different aspects or properties of a stimulus, different task rules, or different effectors, frequently relying on retrieval from working memory. Multitasking (dual-tasking) requires subjects to perform several tasks concurrently, putting a strain on information processing resources.

\section{Task switching}

Studies of cognitive switching commonly report a decline in older age (Wecker et al., 2005), although there is also some evidence to the contrary (Logie et al., 2004; Della Sala et al., 2010). Age effects have been seen to diminish after extensive training on a switching task (Kramer et al., 1999), even when requiring switching between four different tasks (Buchler et al., 2008). Kray and Lindenberger (2000) differentiate between mixing and switch costs. Switch costs refer to increased latencies and error rates on switch trials compared to non-switch trials. Mixing costs are slower or more error-prone responses occurring when performing non-switch trials in the context of a switch task compared to the context of a single task. Though both types of costs can be reduced by training, mixing costs are suggested to be more compromised by aging than switch costs (Kray and Lindenberger, 2000; Kray et al., 2008), implying that aging especially affects the ability to keep multiple sets in working memory rather than making the shift itself. At the same time, mixing costs are also most sensitive to improvement (Strobach et al., 2011). Kray et al. (2008) found that when subjects verbalized their task cues before shifting to a different task, a reduction of mixing costs was seen especially in older adults compared to younger adults, whereas switch costs did not benefit from verbalizations.

Not only does training in task switching demonstrate enhancement on the task itself, recent studies also show the possibility of near and far transfer. Elderly who have grown up as bilinguals, thus constantly needing to switch between the two languages during their lifetime, are found to have an advantage in inhibitory control compared to monolingual elderly (Bialystok et al., 2004, 2006). Older adults who received training in task switching showed a reduction in mixing and switch costs on a similar switch task (Karbach and Kray, 2009; Karbach et al., 2010), but also displayed reduced interference effects on a Stroop task, and improved spatial and verbal working memory and fluid 
intelligence, in contrast to baseline and to elderly subjects receiving similar, non-switching related training (Karbach and Kray, 2009). In this study, demands were not only on task-set selection, but also on interference control and on goal maintenance, thus requiring use of multiple cognitive control mechanisms in one task. The fact that this intervention led to generalizable learning highlights the importance of engaging multiple mechanisms in training tasks.

\section{Multitasking}

Elderly adults generally experience greater dual-tasking costs compared to young, even when taking age-related general slowing into account (Verhaeghen et al., 2003; Bherer et al., 2005, 2008). Evidence from a recent fMRI study implies that during dual-tasking, elderly are unable to sufficiently disengage from the interruption by the second task and therefore fail to switch back to the appropriate functional network, which causes greater difficulty with dual-tasking (Clapp et al., 2011). Intervention studies show that elderly were able to benefit from dual-task training at the same rate as young. Bherer et al. (2005) trained elderly on a three-week long paradigm where visual identification and auditory discrimination were performed either concurrently or separately. Response latency was reduced in elderly to the same extent as in young adults, and accuracy improvement was even more pronounced among seniors, especially in the concurrent tasks. Near transfer was found on within-modality and crossmodality dual-task costs, and was as large (or larger) in old as in young. Assessment one month later suggested retention of the training effect. A follow-up study using two concurrent visual tasks reported similar training benefits among seniors (Bherer et al., 2008). This implies that improvement of multitasking can occur regardless of whether training consisted of same- or different-modality tasks.

Training on dual-tasking paradigms has also been suggested to transfer considerably to daily-life performance. When elderly and young subjects were trained on a driving simulation, which included a visual attention task and a visuomotor tracking task, elderly decreased their error count and response latencies to a greater extent than young adults (Hahn et al., 2010). On that same note, after computerized training on tasks combining working memory, attention, and manual control, older adults showed significant improvement in simulated driving performance (Cassavaugh and Kramer, 2009), where performance improvements on dual task effects were predictive of later driving performance improvements. Li et al. (2010) demonstrated transfer of visual discrimination multitasking to single- and double-support standing balance. Hence, multitasking interventions show generalization to activities that are directly relevant to elderly.

\section{UPDATING}

Updating, an essential aspect of working memory, refers to monitoring incoming information for its relevance and accordingly adapting the content of working memory storage, and has been linked to activation in frontopolar and dorsolateral prefrontal cortex (Salmon et al., 1996; Van Der Linden et al., 1999). Elderly performing updating tasks invest greater effort than young adults
(Fiore et al., 2012). In one updating task, in which participants updated memory by remembering the smallest item on a list, four age groups (young, young-old, old, and old-old) were compared (De Beni and Palladino, 2004). Performance on this task declined more with increasing age, and older participants suffered more difficulty to suppress intrusions.

Despite age-related deficits, training of this paradigm in elderly has demonstrated opportunities for transfer. Near transfer to block-span performance was found after a 12-week training intervention (Buschkuehl et al., 2008) which included three different updating paradigms. Successful training on updating tasks was also done by Dahlin et al. (2008a,b) who trained older adults on letter-memory updating, which requires keeping a string of letters in working memory and recalling the last four letters upon ending of the task. Elderly displayed increased task performance which was maintained up to 18 months post-training, and training-related activation in striatum compared to controls.

One type of test often used to assess updating is the n-back paradigm, in which participants respond when the current stimulus matches that of $\mathrm{n}$ trials back. N-back tasks have been tested in elderly before, indicating the ability of elderly subjects to perform this task, even with increased working-memory demand (Verhaeghen and Basak, 2005; Van Gerven et al., 2008; Jaeggi et al., 2009). In young adults, training on this paradigm with a dual (visual and auditory) component is implied to lead to far transfer to fluid intelligence (Jaeggi et al., 2008; but see Moody (2009) for a critical evaluation).

To our knowledge, few longitudinal n-back training studies have been conducted in elderly. In one study, young and older adults were trained on a demanding spatial 2-back task (Li et al., 2008) which included blocks of regular spatial updating and trials which additionally required mental rotation. In old and young adults equally, near transfer to a more demanding spatial 3back task and numerical 2-back and 3-back tasks was found. This performance was largely maintained 3 months after posttest. A 3-back spatial task has also been included as part of an effective multimodal training battery (Schmiedek et al., 2010). These results suggest that the older population might benefit from training on n-back tasks as well (although this claim has been contested by Engle and colleagues, see e.g., Shipstead et al., 2010). Further testing of this paradigm, including the possibility of transfer to untrained domains, seems a promising avenue for further research.

\section{INHIBITION}

Inhibition refers to the suppression of thoughts or actions, usually in favor of other thoughts or actions. Inhibition may be at play at various levels: preventing irrelevant sources of information from capturing attention, preventing irrelevant contents of information from entering working memory, pre-empting rash decisions, suppressing impulsive or undesirable actions, or overriding prepotent responses in favor of more appropriate ones. Inhibition at the levels of attention and working memory have been associated with the functionality of frontoparietal systems (Hasher and Zacks, 1988), whereas inhibition in relation to decision-making and action have been linked to the integrity of frontostriatal circuitry (Ridderinkhof et al., 2004). A variety of tasks and tests 
have been proposed to assess inhibitory efficiency in older adults, but many of these tasks (and associated age effects) suffer from problems with task purity, methodological confounds, and other measurement issues that are characteristic of many so-called frontal-lobe tests (Rabbitt et al., 2001). Nonetheless, there appears to be consensus that the ability to inhibit spatial responses is relatively preserved, whereas the ability to actively inhibit prepotent responses shows more robust age effects (Nieuwenhuis et al., 2000; Andres et al., 2008) in the form of reduced inhibitory control over reflexive saccades in the antisaccade task (for review, see Eenshuistra et al., 2004) and a reduced ability to interrupt actions that have already been initiated in the stop task (Williams et al., 1999).

To our knowledge, although a number of brain training studies have included inhibitory tasks in one form or other, no studies have focused specifically and systematically on whether the effects of old age on inhibitory efficiency can be remedied by training. One study reported that inhibitory skills can be trained in children (Thorell et al., 2009). That such training is feasible at least in principle in adults was demonstrated in a recent study with young adults, whose Go/NoGo proficiency improved after only a single and brief training session (Manuel et al., 2010). Evidence ubiquitously suggests age-related increases in susceptibility to interference in the Stroop task. Training studies have examined the effects of Stroop-task training in elderly, reporting performance improvement but no transfer (Dulaney and Rogers, 1994; Davidson et al., 2003). Unfortunately, the task impurity that characterizes the Stroop task (involving perceptual interference and task maintenance demands in addition to response inhibition, presumably leaving little age-related variance left to be explained by the latter) renders this task less suitable for studying the effects of age and training on inhibitory control. Using relatively more pure measures of response inhibition, age trends in inhibitory efficiency were reported in the Simon task (Maylor et al., 2011). Whether and to what extent these measures may benefit from training remains to be explored. Likewise, we are not aware of brain training studies using antisaccade tasks. Our own work has shown that antisaccade performance in the elderly may be improved considerably by motivational factors (such as reward anticipation; Harsay et al., 2010), suggesting that there may be substantial space for improvement using brain training.

\section{SUMMARY}

In sum, from the studies reviewed above it becomes evident that continuous training on cognitive control-based paradigms may not only lead to enhanced performance on the trained task, but may on occasion also extend to other, untrained, domains. This holds true in particular for tasks that capitalize in one way or other on cognitive flexibility, especially apparent in task switching paradigms (for instance, Karbach and Kray, 2009). Ideally, therefore, tasks should call on flexibility. They should engage multiple mechanisms of cognitive control at the same time, e.g., keeping a number of items in memory, shifting attention between tasks, inhibiting irrelevant stimuli while responding to another, and updating the memory trace. Subjects are thereby forced to divide their attention over a number of multimodal stimuli, creating a general state of alertness and preparedness for upcoming events that is likely to be generalized to functioning on other, nonrelated tasks.

\section{ADDITIONAL PERSPECTIVES}

A number of modalities that appear especially relevant to cognitive aging might be effectively trainable in this population, although so far there has been little investigation into these perspectives. First of all, decision-making and learning from mistakes are affected in old age, and a number of ideas to aid in dealing with these deficits are recounted below. Second, novelty can be an important key to add to training benefit in two separate ways, which will be argued here. Finally, memory strategy training has been shown to be effective in the elderly population. Though no evidence of far transfer currently exists, suggestions are given for ways to test this more thoroughly.

\section{DECISION-MAKING}

One domain that is also affected by age is decision-making and decision-learning (Brown and Ridderinkhof, 2009; Mohr et al., 2010). Older adults have more difficulty with stimulusreward learning, taking longer to reach a criterion and displaying impaired feedback learning (Schmitt-Eliassen et al., 2007). Older individuals are generally more proficient at avoidance-learning compared to incentive-learning; they exhibit a bias to choose to avoid negative outcomes rather than gaining positive outcomes, thought to result from age-related loss of dopamine (Frank and Kong, 2008). Studies assessing learning abilities in elderly using the Iowa Gambling Task, where one needs to learn to choose cards from the most beneficial deck to optimize reward, have resulted in mixed findings. Some suggest that elderly do not sufficiently learn to pick the most profitable deck (Fein et al., 2007); others find that this impaired learning only applies to a subgroup of elderly (Denburg et al., 2006), illustrating the individual variation in this population. Increased age has also been found to be related to greater reward-related risk-taking (Cavanagh et al., 2012), in particular when learning has led to risk-avoiding behavior (Mata et al., 2011). Furthermore, it seems that elderly display an alternate activation pattern of the ventral striatum during reward anticipation and delivery. Although in elderly the ventral striatum is engaged to represent reward value, this region often fails to show activation when anticipating reward (Schott et al., 2007). Unlike in young, there is a failure to activate the insula during loss prediction (Samanez-Larkin et al., 2007), demonstrating their ability to process reward value but an inability to engage the necessary regions during anticipation.

Delay discounting refers to the preference for more immediate, smaller rewards relative to later, larger rewards. The ability to forego an immediate reward in favor of some future interest (a crucial aspect of decision-making, also in a variety of daily-life decisions) has been associated with striatal dopamine; hence, one might expect the proficiency of delay discounting to decline with age. Results are mixed and contradictory, however (e.g., Chao et al., 2009; Reimers et al., 2009; Jimura et al., 2011; Löckenhoff et al., 2011), preventing us from drawing firm conclusions at this stage.

Given these patterns of aging-related deficits in decisionmaking and decision-learning, and the importance for 
independently functioning elderly to be able to make essential decisions for themselves, one might expect decision-learning to be included in one way or other in brain training programs. We are not aware, however, of any training studies in the realm of outcome optimization. Yet, the success of such training seems feasible. For instance, anticipation of a rewarding outcome has been shown to motivate successful optimization strategies in elderly (Denburg et al., 2006; Harsay et al., 2010). Along another avenue, older decision makers appear to base their decisions on less information than younger decision makers, since this leads to only small losses in decision quality (Mata and Nunes, 2010). Thus, brain training programs might focus on training the ability to select target information economically. Moreover, aging appears to be associated specifically with deficits in rule-based decision-making processes (Mata et al., 2011) suggesting that training protocols can be targeted to learning simple and (as learning progresses) more complex decision rules in choice games, and to learning that rule-based decisions lead to favorable outcomes more often than, for instance, similarity-based decisions.

\section{NOVELTY}

Cognitive processes can be more adequately stimulated by including the important ingredient of novelty: an item, task, or activity that is unfamiliar and has not yet become subject to automatization. There are two ways in which novelty inclusion can benefit training studies and lead to reduced cognitive decline: to improve performance on existing tasks by direct inclusion of novel stimuli within training tasks, and to improve performance on new tasks by creating novel experiences and activities as the core of training. Along these lines, besides inducing novelty within tasks, an enriched environment can offer a similar effect, challenging the neuronal system to develop or protecting it from negative aging influences, as has been shown in aging animals (Winocur, 1998; Kempermann et al., 2002) as well as humans (Karp et al., 2006).

One type of intervention may contribute to protracted cognitive decline by adding features of novelty to an existing task. Stimulus repetition often leads to a decrease in neural activity as a result of more efficient neural processing (Ranganath and Rainer, 2003); by contrast, inclusion of novel stimuli is often followed by an increase in activity, and has been demonstrated to enhance synaptic plasticity, thereby posing an advantage for interventions.

Neuromodulation is believed to play an important role in the encoding of novel information into memory. Acetylcholine as well as norepinephrine have been shown to facilitate consolidation of novel stimuli by increasing the firing rate and enhancing responses to stimuli. This is also illustrated by administration of anticholinergics, which attenuates electrophysiological and hemodynamic expression of the effects of novel compared to familiar stimuli (Ranganath and Rainer, 2003).

Düzel et al. (2010) argue that novelty processing in the brain can enhance plasticity by boosting dopamine to benefit learning and memory and allow long-term consolidation to take place within the hippocampus. Dopaminergic neuromodulation occurs during and after exposure to novel stimuli, facilitating long-term potentiation and leading to consolidated synaptic plasticity. The authors suggest an integrative model of exploratory drive and neuronal plasticity to explain the connections between dopamine, novelty and plasticity, specifically in old age. According to this model, an individual is motivated to perform exploratory behavior following novelty expectation. As dopaminergic neuromodulation is subject to deterioration with increased age, elderly generally receive less reinforcement from novelty and would naturally tend less towards seeking novel stimuli in their environments, thereby creating less opportunity for plasticity and learning to take place. Although older adults benefit less from inclusion of novelty compared to younger individuals, they are still thought to benefit from a boost of dopamine to create a better learning opportunity.

Few studies have examined the role of novelty in protracting neurocognitive decline directly. One line of studies used randomized trials to investigate training abilities in elderly participants by teaching and training skills in novel activities. For instance, Bugos et al. (2007) explored individual piano instruction as a cognitive intervention in the elderly population. A group of musically naïve elderly subjects were given weekly piano and music theory lessons and were required to practice independently for $3 \mathrm{~h}$ each week for a period of 6 months. Compared to a control group who received no training, transfer of training was seen on Digit Symbol (a subtest of the WAIS) and the Trail Making Test, suggesting that music training led to improvement of concentration, attention, and planning abilities. Likewise, Boyke et al. (2008) studied a group of healthy elderly learning to juggle. They were given 3 months to learn and practice, and MRI scans were made directly before and after training, and 3 months after training had ended. Changes included gray-matter increases in brain areas responsible for processing of complex visual movement, and did not appear in the control group. These structural changes occurred even in individuals who were not able to perform satisfactorily at the time of testing, suggesting neuronal plasticity even among seniors who take longer to learn a new ability. A follow-up study using a small sample of young adults suggests that these structural changes might be produced by learning of the novel skill per se, with little further contribution from the amount of practice or the eventual quantitative increases in performance (Driemeyer et al., 2008). Unfortunately, the latter study did not make use of any control group, so that further investigation of how much practice is needed to produce structural changes remains necessary. However, so far it seems that learning novel activities can lead to improvement and transfer to other tasks, as well as to structural brain changes in old age.

Novel and challenging experiences during the lifetime are thought to also benefit cognition in old age. Neurocognitive aging processes may speed up when individuals no longer engage in work-related or social activities and hence withdraw from stimulating environments that frequently present novel stimuli or challenges (Aichberger et al., 2010; Roberts et al., 2011), possibly through weakening of neuronal connections (Cerella and Hale, 1994). Actively taking part in cognitively challenging activities is thought to function as a protective factor against cognitive decline and even decreases the risk of development of age-related diseases (Karp et al., 2006; Bialystok et al., 2007; Yaffe et al., 2009). There appears to be a strong connection between involvement in complex and challenging work during early adult life, and subsequent 
intellectual functioning in old age (Schooler et al., 1999). Job complexity is also believed to offer a protective factor against dementia (Potter et al., 2007). However, these studies were not able to control for confounds, leaving open the possibility that the mentioned relationship between complexity and novelty in early life and functioning in older age is bidirectional, that is, although a stimulating work environment probably affects workers' cognitive wellbeing and challenges them to broaden their intellectual horizons, individuals' already existing intellectual functioning also causes people to choose for more challenging and intellectual vocations to match their abilities. Bosma et al. (2003) analyzed the protective effects of work load on later cognitive functioning longitudinally, but controlled for a number of confounds including education and baseline intellectual abilities. When adjusting for these factors, individuals with higher workload showed a greatly decreased risk of developing later cognitive impairment.

These outcomes emphasize the promise held by the training of novel skills or the inclusion of novel stimuli in training programs. Novelty not only primes the neuronal system to prepare for learning, but the addition of continuous novel stimulation itself, be it in a standardized task or in learning a new ability, also helps build new connections and could add to individuals' motivation, providing important benefits for maintaining cognitive wellness.

\section{MEMORY STRATEGIES}

Several aspects of working memory training, such as updating, have been reviewed above. One could argue that these concentrate on training processes. This approach must be distinguished from developing new memory strategies (Kliegel and Bürki, 2012). These have a long history (Yates, 1966) and there is a considerable body of literature that demonstrates their effectiveness (Higbee, 1993). This suggests that they may also be applicable as a successful form of brain training in the elderly. Memory strategies are often taught as part of a more general memory training, which can range from learning a simple mnemonic strategy to extensive practice with a wide range of memory techniques. Rebok et al. (2007) reviewed almost 300 memory training studies with older adults using explicit criteria to judge whether the improvements due to a certain type of training could be considered evidence-based. According to the criteria, a type of training showed a beneficial effect if more than $50 \%$ of the outcome measures were both statistically significant between-group treatment effects (within-group studies were compared with baseline) and had effect sizes of at least 0.20 . Evidence criteria for a certain type of memory training stipulated, furthermore, that there be at least two such studies with beneficial effects, with a minimum of 30 participants in total. Of the 218 studies considered by Rebok et al. (2007), 39 studies contribute support to 16 types of memory training which effects could be considered evidence-based. In particular, studies involving instruction in multiple mnemonic techniques led to lasting improvements (e.g., Stigsdotter and Bäckman, 1989; Hill et al., 1990; Ball et al., 2002; Dunlosky et al., 2003). Also, training specific strategies such as visual memory support (Sharps and Price-Sharps, 1996), the story mnemonic (Hill et al., 1991), and the classic loci method (Kliegl et al., 1989; Hill et al., 1991) gave significant results that qualified on the evidence-based criteria. Rebok et al. (2007, p. 54) conclude that these findings suggest "...that there are potentially several evidence-based options for older adults who wish to improve their memory and reduce memory problems."

Whereas there are clear benefits from certain types of strategybased memory training, it is not clear at this point whether they also give rise to long-term benefits. Zelinski (2009), for example, concludes that training specific mnemonic strategies in isolation does not seem to lead to far transfer. Few studies have attempted extensive training on a variety of strategies. One example is a recent study by Craik et al. (2007) who instructed 49 older adults in a variety of mnemonic strategies (among other aspects of the training). The instruction sessions encouraged subjects to practice and find their own optimal combination, but there was no formal guidance, nor was there a computerized training that supported the optimization process. Craik et al. (2007) found no improvements on primary memory or working memory, but they did find a lasting improvement on episodic memory. A limitation of this study is that as part of the design, half the subjects had to wait three months after entering the study and initial orientation before they received the majority of the training. As the authors remark, this led a loss of motivation in the late group and hence to a loss of power in the experiment.

Craik et al. (2007) allowed subjects to control and combine their optimal strategies. Complete self-generation of strategies is thought to be a particularly effective method. In Lustig and Flegal (2008), subjects were shown individually presented words to encode and remember as well as possible. They were assigned to either a condition in which they learned to use a specific encoding strategy, or a condition in which they could choose their own strategy. Transfer to an unrelated task was found only in the strategy choice condition. This suggests that it is most beneficial to engage and train preserved albeit dormant functions in elderly by letting them initiate their own optimal strategy, in order for deep encoding processes to occur to lead to more generalizable results. Derwinger et al. (2005) also found that in older individuals, self generation of strategies is most optimal. Although subjects using learned mnemonic strategies and selfgenerated strategies to memorize four-digit numbers retained the same amount of information on the short-term memory tasks, long-term recollection was better in the strategy-choice condition.

We find evidence for beneficial effects of strategy-based memory training, though successful studies that yield far transfer are currently lacking. We suggest the use of a computerized approach in order to ensure that strategies are indeed being trained and to help subjects in their development and application.

\section{SUMMARY}

A number of additional modalities have been discussed that could potentially be used to add to effective training purposes, though more research is needed to confirm this. Given the agingrelated deficits in decision-making and decision-learning, aspects of decision-learning might be included in training programs by focusing, for instance, on reward anticipation or rule-based decision-making. Novelty seems to be an important factor for more lasting effects of brain training, especially in elderly, and 
inclusion of novel stimuli or tasks could motivate elderly to invest more effort and energy into learning. In strategy-based memory training, most success is to be expected from studies that employ a variety of memory strategies, allowing considerable freedom to select optimal combinations of these, and include extensive practice.

\section{THE INDIVIDUAL PICTURE VARIATION WITHIN THE AGING POPULATION}

One major caveat in much of the literature on brain training research concerns individual differences in the aging population. First, aging studies frequently use a comparison of retired, independent elderly of various different backgrounds to young adults, often psychology students. Besides the fact that both groups are often recruited from different sources, which impacts the validity of these studies, students and retirees are likely to differ in several other ways than age alone (e.g., length and type of education or exposure to technology), making it more difficult to attribute any observed differences directly to age-related decline and skewing the implications of age-related cognitive decline as derived from these results.

Second, and perhaps even more important, in the current literature elderly individuals are often measured as a group, without paying attention to the existing and evident differences between individuals. Elderly are likely to differ more from each other than young adults do. Genetic and environmental, traumatic and advantageous influences have a lifelong effect on each person's brain and behavior (Christensen et al., 1999; Bialystok et al., 2004; Lindenberger et al., 2008), thus exaggerating inter-individual variability as the individuals grow older. To draw conclusions on trainability of a certain task based on the mean performance of a group of elderly does little justice to individuals' strengths and weaknesses and paints a picture of the potential effects of training that is not sufficiently representative as it tends to blend all nuances in the color palette into a single shade of grey. Certain individuals might have a larger rate of cognitive decline than others, while yet others might show little decline at all. Variability in cognitive performance may result also from, for instance, illness or depression (Christensen et al., 1999). Such variation is likely to cause inconsistencies within training studies, resulting in poor conclusions about the success of certain interventions or inaccurate rejections of training paradigms that could be helpful to some, but might not work for most. Some studies that attempt to take individual variation in baseline parameters (such as workingmemory capacity or general processing efficiency) into account even arrive at the conclusion that age-related differences in cognitive performance can be reduced to age-related differences in these baseline parameters (Eenshuistra et al., 2004; Della Sala et al., 2010). Current brain training research is based on the question whether a paradigm is either successful or unsuccessful; instead, we might profit more from asking for whom the training works, and how these individuals vary from the rest, in terms of behavioral and neuroimaging measures. Each person is likely to benefit from different training approaches (Yaffe et al., 2009). Some might benefit more from some tasks than from others, and some people might need more intensive training, whereas others lose motivation because training tasks do not pose enough of a challenge. Adaptive training is tailored to the needs and abilities of the individual, increasing difficulty levels as one gets better and decreasing them as more errors occur. Adding an adaptive component to the training is therefore crucial to allow people to train at their own level and keep each person challenged and motivated. Most training studies do not pay attention to this aspect, though some have (Mahncke et al., 2006; Ball et al., 2007; Smith et al., 2009). Lustig and Flegal (2008) showed that memory training performance was most effective when subjects were allowed to explore and initiate their own latent optimal strategy. It seems crucial that, during training, subjects should experience success yet stay challenged enough to increase performance. Finally, the gains associated with working-memory training were found to depend on genotypes related to the expression of dopamine in the substantia nigra (Bellander et al., 2011).

For those individuals who benefit less from brain training, alternative intervention possibilities can be explored. An important challenge, then, lies in identifying predictors of individual differences in trainability. These predictors could consist of certain neurocognitive test results, but importantly also of data on individual neural hard-wiring: neuromodulation, regional brain volume, structural and functional connectivity, or functional activation patterns. In the next section we assess in more detail the benefits that covariance-based neuroimaging techniques might provide in helping us understand individual differences in cognitive decline and trainability.

\section{IMAGING INDIVIDUAL DIFFERENCES}

Recent progress has advanced our insight in functional and structural alterations in healthy aging as related to individual performance differences (independent of baseline structural volume or age per se). For instance, BOLD (de)activation patterns can illustrate associations with reduced or retained performance. Two groups of elderly who had shown similar IQ at age 11 but whose IQ scores diverged at age 70 were compared, thus forming a group of cognitive "sustainers" and "decliners" (Waiter et al., 2008). fMRI data of the elderly group was subsequently compared to that of a young subject group. Whereas neural activation for the sustainer group did not vary from the brain regions active in young during a visual inspection task, decliners showed deactivation in a number of these areas; neural activation was found to predict individual preservation of complex reasoning skills. Similarly, in the memory domain, increased neural activation during an emotional word judgment task was observed in young adults and in a subgroup of elderly with normal performance, but not in elderly with declined memory performance (Daselaar et al., 2003). Variance in episodic recall performance has also been linked to hippocampal volume and activation change (Persson et al., 2006). Evidence that fMRI results could be applied to predict clinical cognitive decline comes from O'brien et al. (2010) demonstrating that individuals without signs of dementia at baseline but with a Clinical Dementia Rating (CDR) score of 0.5 showed a decline in hippocampal activity on an associative memory task over a period of 2 years, whereas activation patterns of those with a CDR score of 0 remained the same.

At the structural level, more complex sulcal folding was correlated with higher maintenance of cognitive processing speed 
(Kochunov et al., 2010; Liu et al., 2011). Measurement of postsynaptic markers has also been related to cognitive performance in elderly. Using PET, increased caudate dopamine uptake was found to be related to higher working memory capacity while dopamine uptake in putamen was connected to increased motor speed (Landau et al., 2009). D2 receptor binding has been found to account for differences in individual cognitive performance more than age did (Bäckman et al., 2000), especially in motor functions and tasks dependent on frontal brain areas (Volkow et al., 1998). These and more recent findings suggest that individual age-related changes in prefrontal and striatal dopaminergic systems underlie performance decline (e.g., Bennett et al., 2010; Klostermann et al., 2011; Samanez-Larkin et al., 2011).

In future studies, combining some of these imaging techniques to examine the individual differences in trainability among elderly could lead to important insights about which individuals do or do not benefit from specific types of training, so that alternative interventions can be considered. For instance, striatal volume pretraining was found to account for improvement of young adults on a strategy video game (Erickson et al., 2010). Nucleus accumbens volume predicted success during early training while larger dorsal striatum volume was associated with improvement of performance throughout the training. To our knowledge, similar neuroimaging perspectives examining predictors of individual training success have currently not been investigated in the elderly brain training literature. In one study (Engvig et al., 2011), elderly subjects were scanned before and after memory training using DTI to measure changes in white matter tracts, and found an increase in FA in the training group, demonstrating the sensitivity of DTI to display differences in white matter over a period of 10 weeks. Moreover, individual memory improvement was significantly related to strength of FA change. This illustrates the possibility to show individual differences in training success in elderly, allowing future research to explore the potential of this methodology.

\section{REFERENCES}

Aichberger, M. C., Busch, M. A., Reischies, F. M., Ströhle, A., Heinz, A., and Rapp, M. A. (2010). Effect of physical inactivity on cognitive performance after 2.5 years of follow-up: longitudinal results from the survey of health, ageing, and retirement (SHARE). GeroPsych. J. Gerontopsychol. Geriatr. Psychiatry 23, 7.

Andres, P., Guerrini, C., Phillips, L. H., and Perfect, T. J. (2008). Differential effects of aging on executive and automatic inhibition. Dev. Neuropsychol. 33, 101-123.

Bäckman, L., Ginovart, N., Dixon, R. A., Wahlin, T. B., Wahlin, A., Halldin, C., and Farde, L. (2000). Age-related cognitive deficits mediated by changes in the striatal dopamine system. Am. J. Psychiatry $157,635-637$.

Bäckman, L., Nyberg, L., Lindenberger, U., Li, S. C., and Farde, L. (2006).
The correlative triad among aging, dopamine, and cognition: current status and future prospects. Neurosci. Biobehav. Rev. 30, 791-807.

Ball, K., Berch, D. B., Helmers, K. F., Jobe, J. B., Leveck, M. D., Marsiske, M., Morris, J. N., Rebok, G. W., Smith, D. M., Tennstedt, S. L., (2002). Effects of cognitive training interventions with older adults - A randomized controlled trial. JAMA 288, 2271-2281.

Ball, K., Edwards, J. D., and Ross, L. A. (2007). The impact of speed of processing training on cognitive and everyday functions. J. Gerontol. B Psychol. Sci. Soc. Sci. 62, 19-31.

Bellander, M., Brehmer, Y., Westerberg, H., Karlsson, S., Fürth, D., Bergman, O., Eriksson, E., and Bäckman, L. (2011). Preliminary evidence that allelic variation in the LMX1A gene influences training-related Unverzagt, F. W., and Willis, S. L.

\section{CONCLUSION}

In pursuit of successful cognitive interventions, different training activities have been used to preserve and improve cognitive functioning in the aging population. Despite ample evidence showing that improvement is indeed possible, results are not consistently positive. We have sketched a number of ways in which future interventions could promote robust and generalized preservation of function. In order to attain long-term retention and transfer, plasticity is key. Cognitive processes can be more adequately stimulated by including the important ingredient of variability: requiring subjects to integrate cognitive functions rather than training separate mechanisms. Because cognitive domains are behaviorally and neurologically intertwined, maximal profit is reached if not just one, but multiple functions are engaged with the tasks at hand. We therefore suggest that brain training tasks be multimodal, tax cognitive flexibility, and capitalize on novelty to stimulate plasticity to the highest extent. These properties tend not to be naturally included in older adults' daily activities. Yet, this very fact points out the relevance of using these properties in this population in order to offer an optimally challenging environment. A successful brain training program should preferably include a range of different tasks to engage a multitude of functions, as well as continually offer something new in order for the neuronal system to remain challenged and to create possibilities of maximum enhancement in this population. We further argue for the importance of paying attention to individual differences in training benefit. This is possible both by incorporating adaptive elements into training, thus allowing each individual to improve at their own pace, according to their already existing abilities and in tune with their individual and momentary motivational needs. Finally, we recommend the application of innovative covariance-based neuroimaging methods to studies of brain training to investigate neural predictors of individual differences in trainability.

working memory improvement. Neuropsychologia 49, 1938-1942.

Bennett, I. J., Madden, D. J., Vaidya, C. J., Howard, J. H. Jr., and Howard, D. V. (2010). White matter integrity correlates of implicit sequence learning in healthy aging. Neurobiol. Aging 32, 2317.e1-2317.e12.

Bherer, L., Kramer, A. F., Peterson, M. S., Colcombe, S., Erickson, K., and Becic, E. (2005). Training effects on dual-task performance: are there age-related differences in plasticity of attentional control? Psychol. Aging 20, 695-709.

Bherer, L., Kramer, A. F., Peterson, M. S., Colcombe, S., Erickson, K., and Becic, E. (2008). Transfer effects in task-set cost and dualtask cost after dual-task training in older and younger adults: further evidence for cognitive plasticity in attentional control in late adulthood. Exp. Aging Res. 34, 188-219.
Bialystok, E., Craik, F. I., and Freedman, M. (2007). Bilingualism as a protection against the onset of symptoms of dementia. Neuropsychologia 45, 459-464.

Bialystok, E., Craik, F. I., Klein, R., and Viswanathan, M. (2004). Bilingualism, aging, and cognitive control: evidence from the Simon task. Psychol. Aging 19, 290-303.

Bialystok, E., Craik, F. I., and Ryan, J. (2006). Executive control in a modified antisaccade task: effects of aging and bilingualism. J. Exp. Psychol. Learn. Mem. Cogn. 32, 1341-1354.

Bosma, H., Van Boxtel, M. P., Ponds, R. W., Houx, P. J., Burdorf, A., and Jolles, J. (2003). Mental work demands protect against cognitive impairment: MAAS prospective cohort study. Exp. Aging Res. 29, 33-45.

Boyke, J., Driemeyer, J., Gaser, C., Buchel, C., and May, A. (2008). Training-induced brain structure 
changes in the elderly. J. Neurosci. 28, 7031-7035.

Brown, S. B., and Ridderinkhof, K. R. (2009). Aging and the neuroeconomics of decision making: a review. Cogn. Affect. Behav. Neurosci. 9, 365-379.

Buchler, N. G., Hoyer, W. J., and Cerella, J. (2008). Rules and more rules: the effects of multiple tasks, extensive training, and aging on task-switching performance. Mem. Cognit. 36, 735-748.

Bugos, J. A., Perlstein, W. M., McCrae, C. S., Brophy, T. S., and Bedenbaugh, P. H. (2007). Individualized piano instruction enhances executive functioning and working memory in older adults. Aging Ment. Health 11, 464-471.

Buschkuehl, M., Jaeggi, S. M., Hutchison, S., Perrig-Chiello, P., Däpp, C., Müller, M., Breil, F., Hoppeler, H., and Perrig, W. J. (2008). Impact of working memory training on memory performance in old-old adults. Psychol. Aging 23, 743 .

Cassavaugh, N. D., and Kramer, A. F. (2009). Transfer of computerbased training to simulated driving in older adults. Appl. Ergon. 40, 943-952.

Cavanagh, J. F., Neville, D., Cohen, M. X., Van de Vijver, I., Harsay, H., Watson, P., Buitenweg, J. I., and Ridderinkhof, K. R. (2012). Individual differences in risky decision-making among seniors reflect increased reward sensitivity. Front. Neurosci. 6:111. doi: 10.3389/fnins.2012.00111

Cerella, J., and Hale, S. (1994). The rise and fall in information-processing rates over the life span. Acta Psychol. (Amst.) 86, 109-197.

Chao, L. W., Szrek, H., Pereira, N. S., and Pauly, M. V. (2009). Time preference and its relationship with age, health, and survival probability. Judgm. Decis. Mak. 4, 1.

Christensen, H., Mackinnon, A. J., Korten, A. E., Jorm, A. F., Henderson, A. S., Jacomb, P., and Rodgers, B. (1999). An analysis of diversity in the cognitive performance of elderly community dwellers: individual differences in change scores as a function of age. Psychol. Aging 14, 365-379.

Clapp, W. C., Rubens, M. T., Sabharwal, J., and Gazzaley, A. (2011). Deficit in switching between functional brain networks underlies the impact of multitasking on working memory in older adults. Proc. Natl. Acad. Sci. U.S.A. 108, 7212-7217.

Colcombe, S. J., Erickson, K. I., Raz, N., Webb, A. G., Cohen, N. J.,
McAuley, E., and Kramer, A. F. (2003). Aerobic fitness reduces brain tissue loss in aging humans. J. Gerontol. A Biol. Sci. Med. Sci. 58, 176-180.

Colcombe, S. J., Erickson, K. I., Scalf, P. E., Kim, J. S., Prakash, R., McAuley, E., Elavsky, S., Marquez, D. X., Hu, L., and Kramer, A. F. (2006). Aerobic exercise training increases brain volume in aging humans. J. Gerontol. A Biol. Sci. Med. Sci. 61, 1166-1170.

Craik, F. I. M., Winocur, G., Palmer, H., Binns, M. A., Edwards, M., Bridges, K., Glazer, P., Chavannes, R., and Stuss, D. T. (2007). Cognitive rehabilitation in the elderly: effects on memory. J. Int. Neuropsychol. Soc. $13,132-142$.

Dahlin, E., Bäckman, L., Neely, A. S., and Nyberg, L. (2009). Training of the executive component of working memory: subcortical areas mediate transfer effects. Restor. Neurol. Neurosci. 27, 405-419.

Dahlin, E., Neely, A. S., Larsson, A., Bäckman, L., and Nyberg, L. (2008a). Transfer of learning after updating training mediated by the striatum. Science 320, 1510-1512.

Dahlin, E., Nyberg, L., Bäckman, L., and Neely, A. S. (2008b). Plasticity of executive functioning in young and older adults: immediate training gains, transfer, and long-term maintenance. Psychol. Aging 23, 720-730.

Daselaar, S. M., Veltman, D. J., Rombouts, S. A., Raaijmakers, J. G., and Jonker, C. (2003). Neuroanatomical correlates of episodic encoding and retrieval in young and elderly subjects. Brain $126,43-56$

Davidson, D. J., Zacks, R. T., and Williams, C. C. (2003). Stroop interference, practice, and aging. Neuropsychol. Dev. Cogn. B Aging Neuropsychol. Cogn.10, 85-98.

De Beni, R., and Palladino, P. (2004). Decline in working memory updating through ageing: intrusion error analyses. Memory 12, 75-89.

Della Sala, S., Foley, J. A., Beschin, N., Allerhand, M., and Logie, R. H. (2010). Assessing dual-task performance using a paper-and-pencil test: normative data. Arch. Clin. Neuropsychol. 25, 410-419.

Denburg, N. L., Recknor, E. C., Bechara, A., and Tranel, D. (2006). Psychophysiological anticipation of positive outcomes promotes advantageous decision-making in normal older persons. Int. J. Psychophysiol. 61, 19-25.

Derwinger, A., Stigsdotter Neely, A., Macdonald, S., and Bäckman, L.
(2005). Forgetting numbers in old age: strategy and learning speed matter. Gerontology 51, 277-284.

Driemeyer, J., Boyke, J., Gaser, C., Buchel, C., and May, A. (2008). Changes in gray matter induced by learning-revisited. PLOS ONE 3:e2669. doi: 10.1371/journal.pone. 0002669

Dulaney, C. L., and Rogers, W. A. (1994). Mechanisms underlying reduction in Stroop interference with practice for young and old adults. J. Exp. Psychol. Lear. Mem. Cogn. 20, 470

Dunlosky, J., Kubat-Silman, A. K. and Hertzog, C. (2003). Training monitoring skills improves older adults' self-paced associative learning. Psychol. Aging 18, 340-345.

Düzel, E., Bunzeck, N., Guitart-Masip M., and Düzel, S. (2010). NOveltyrelated motivation of anticipation and exploration by dopamine (NOMAD): implications for healthy aging. Neurosci. Biobehav. Rev. 34, 660-669.

Eenshuistra, R. M., Ridderinkhof, K. R., and Molen, M. W. (2004). Age-related changes in antisaccade task performance: inhibitory control or working-memory engagement? Brain Cogn. 56, 177-188.

Engvig, A., Fjell, A. M., Westlye, L. T., Moberget, T., Sundseth, O., Larsen, V. A., and Walhovd, K. B. (2011). Memory training impacts short-term changes in aging white matter: a longitudinal diffusion tensor imaging study. Hum. Brain Mapp. doi: 10.1002/hbm.21370. [Epub ahead of print].

Erickson, K. I., Boot, W. R., Basak, C., Neider, M. B., Prakash, R. S., Voss, M. W., Graybiel, A. M., Simons, D. J., Fabiani, M., Gratton, G., and Kramer, A. F. (2010). Striatal volume predicts level of video game skill acquisition. Cereb. Cortex 20, 2522-2530.

Fein, G., McGillivray, S., Finn, P., Band, G., Ridderinkhof, K., Segalowitz, S., Bechara, A., Damasio, A., Damasio, H., and Anderson, S. (2007). Older adults make less advantageous decisions than younger adults: cognitive and psychological correlates. J. Int. Neuropsychol. Soc. 13, 480-489.

Fiore, F., Borella, E., Mammarella, I. C., and De Beni, R. (2012). Age differences in verbal and visuo-spatial working memory updating: evidence from analysis of serial position curves. Memory 20, 14-27.

Fisk, J. E., and Sharp, C. A. (2004). Agerelated impairment in executive functioning: updating, inhibition, shifting, and access. J. Clin. Exp. Neuropsychol. 26, 874-890.

Frank, M. J., and Kong, L. (2008). Learning to avoid in older age. Psychol. Aging 23, 392-398.

Hahn, M., Falkenstein, M., and WildWall, N. (2010). Age-related performance differences in compensatory tracking under a dual task condition. Occup. Ergon. 9, 75-86.

Harsay, H. A., Buitenweg, J. I. V., Wijnen, J. G., Guerreiro, M. J. S., and Ridderinkhof, K. R. (2010). Remedial effects of motivational incentive on declining cognitive control in healthy aging and Parkinson's disease. Front. Aging Neurosci. 2:144. doi: 10.3389/fnagi. 2010.00144

Harsay, H. A., Cohen, M. X., Reneman, L., and Ridderinkhof, K. R. (2011) How the aging brain translates motivational incentive into action: the role of individual differences in striato-cortical white matter pathways. Dev. Cogn. Neurosci. 1, 530-539.

Hasher, L., and Zacks, R. T. (1988). Working memory, comprehension, and aging: A review and a new view. Psychol. Learn. Motiv. 22, 193-225.

Hertzog, C., Kramer, A. F., Wilson, R. S., and Lindenberger, U. (2008). Enrichment effects on adult $\operatorname{cog}$ nitive development. Psychol. Sci. Public Interest $9,1$.

Higbee, K. L. (1993). Your Memory: How it Works and How to Improve it. New York, NY: Paragon house.

Hill, R. D., Allen, C., and McWhorter, P. (1991). Stores as a mnemonic aid for older learners. Psychol. Aging 6, 484-486.

Hill, R. D., Storandt, M., and Simeone, C. (1990). The effects of memory skills training and incentives on free recall in older learners. J. Gerontol. $45,227-232$.

Jaeggi, S. M., Buschkuehl, M., Jonides, J., and Perrig, W. J. (2008) Improving fluid intelligence with training on working memory. Proc. Natl. Acad. Sci. U.S.A. 105, 6829.

Jaeggi, S. M., Schmid, C., Buschkuehl, M., and Perrig, W. J. (2009). Differential age effects in loaddependent memory processing. Neuropsychol. Dev. Cogn. B Aging Neuropsychol. Cogn. 16, 80-102.

Jimura, K., Myerson, J., Hilgard, J. Keighley, J., Braver, T. S., and Green, L. (2011). Domain independence and stability in young and older adults' discounting of delayed rewards. Behav. Processes 87 , 253-259.

Karbach, J., and Kray, J. (2009). How useful is executive control training? Age differences in near and far 
transfer of task-switching training. Dev. Sci. 12, 978-990.

Karbach, J., Mang, S., and Kray, J. (2010). Transfer of task-switching training in older age: the role of verbal processes. Psychol. Aging 25, 677-683.

Karp, A., Paillard-Borg, S., Wang, H. X., Silverstein, M., Winblad, B., and Fratiglioni, L. (2006). Mental, physical and social components in leisure activities equally contribute to decrease dementia risk. Dement. Geriatr. Cogn. Disord. 21, 65-73.

Kempermann, G., Gast, D., and Gage, F. H. (2002). Neuroplasticity in old age: sustained fivefold induction of hippocampal neurogenesis by long-term environmental enrichment. Ann. Neurol. 52, 135-143.

Kliegel, M., and Bürki, C. (2012). Memory training interventions require a tailor-made approach: commentary on McDaniel and Bugg. J. Appl. Res. Mem. Cogn. 1, 58-60.

Kliegl, R., Smith, J., and Baltes, P. B. (1989). Testing-the-limits and the study of adult age differences in cognitive plasticity of a mnemonic skill. Dev. Psychol. 25, 247-256.

Klostermann, E. C., Braskie, M. N., Landau, S. M., O'neil, J. P., and Jagust, W. J. (2011). Dopamine and frontostriatal networks in cognitive aging. Neurobiol. Aging 33, 623.e615-623.e624.

Kochunov, P., Coyle, T., Lancaster, J., Robin, D. A., Hardies, J., Kochunov, V., Bartzokis, G., Stanley, J., Royall, D., Schlosser, A. E., Null, M., and Fox, P. T. (2010). Processing speed is correlated with cerebral health markers in the frontal lobes as quantified by neuroimaging. Neuroimage 49, 1190-1199.

Kramer, A. F., Hahn, S., and Gopher, D. (1999). Task coordination and aging: explorations of executive control processes in the task switching paradigm. Acta Psychol. (Amst.) 101, 339-378.

Kray, J., Eber, J., and Karbach, J. (2008). Verbal self-instructions in task switching: a compensatory tool for action-control deficits in childhood and old age? Dev. Sci. 11, 223-236.

Kray, J., and Lindenberger, U. (2000). Adult age differences in task switching. Psychol. Aging 15, 126-147.

Landau, S. M., Lal, R., O'neil, J. P., Baker, S., and Jagust, W. J. (2009). Striatal dopamine and working memory. Cereb. Cortex 19, 445-454.

Li, K. Z. H., Roudaia, E., Lussier, M., Bherer, L., Leroux, A., and
McKinley, P. (2010). Benefits of cognitive dual-task training on balance performance in healthy older adults. J. Gerontol. A Biol. Sci. Med. Sci. 65, 1344.

Li, S. C., Schmiedek, F., Huxhold, O., Röcke, C., Smith, J., and Lindenberger, U. (2008). Working memory plasticity in old age: practice gain, transfer, and maintenance. Psychol. Aging 23, 731.

Lindenberger, U., Nagel, I. E., Chicherio, C., Li, S. C., Heekeren, H. R., and Bäckman, L. (2008). Age-related decline in brain resources modulates genetic effects on cognitive functioning. Front. Neurosci. 2, 234-244. doi: 10.3389/neuro.01.039.2008

Liu, T., Wen, W., Zhu, W., Kochan, N. A., Trollor, J. N., Reppermund, S., Jin, J. S., Luo, S., Brodaty, H., and Sachdev, P. S. (2011). The relationship between cortical sulcal variability and cognitive performance in the elderly. Neuroimage 56, 865-873.

Löckenhoff, C. E., O'donoghue, T., and Dunning, D. (2011). Age differences in temporal discounting: the role of dispositional affect and anticipated emotions. Psychol. Aging 26, 274.

Logie, R. H., Cocchini, G., Delia Sala, S., and Baddeley, A. D. (2004). Is there a specific executive capacity for dual task coordination? Evidence from Alzheimer's disease. Neuropsychology 18, 504-513.

Luo, L., and Craik, F. I. (2008). Aging and memory: a cognitive approach. Can. J. Psychiatry 53, 346-353.

Lustig, C., and Flegal, K. E. (2008). Targeting latent function: encouraging effective encoding for successful memory training and transfer. Psychol. Aging 23, 754-764.

Lustig, C., Shah, P., Seidler, R., and Reuter-Lorenz, P. A. (2009). Aging, training, and the brain: a review and future directions. Neuropsychol. Rev. 19, 504-522.

Madden, D. J., Bennett, I. J., and Song, A. W. (2009). Cerebral white matter integrity and cognitive aging: contributions from diffusion tensor imaging. Neuropsychol. Rev. 19, 415-435.

Mahncke, H. W., Connor, B. B., Appelman, J., Ahsanuddin, O. N., Hardy, J. L., Wood, R. A., Joyce, N. M., Boniske, T., Atkins, S. M., and Merzenich, M. M. (2006). Memory enhancement in healthy older adults using a brain plasticity-based training program: a randomized, controlled study. Proc. Natl. Acad. Sci. U.S.A. 103, 12523-12528.
Manuel, A. L., Grivel, J., Bernasconi, F., Murray, M. M., and Spierer, L. (2010). Brain dynamics underlying training-induced improvement in suppressing inappropriate action. J. Neurosci. 30, 13670.

Marks, B. L., Madden, D. J., Bucur, B., Provenzale, J. M., White, L. E., Cabeza, R., and Huettel, S. A. (2007). Role of aerobic fitness and aging on cerebral white matter integrity. Ann. N.Y. Acad. Sci. 1097, 171-174.

Mata, R., Josef, A. K., Samanez-Larkin, G. R., and Hertwig, R. (2011). Age differences in risky choice: a metaanalysis. Ann. N.Y. Acad. Sci. 1235, 18-29.

Mata, R., and Nunes, L. (2010). When less is enough: cognitive aging, information search, and decision quality in consumer choice. Psychol. Aging 25, 289.

Maylor, E. A., Birak, K. S., and Schlaghecken, F. (2011). Inhibitory motor control in old age: evidence for de-automatization? Front. Psychol. 2:132. doi: 10.3389/fpsyg. 2011.00132

Miyake, A., Friedman, N. P., Emerson, M. J., Witzki, A. H., Howerter, A., and Wager, T. D. (2000). The unity and diversity of executive functions and their contributions to complex "Frontal Lobe" tasks: a latent variable analysis. Cogn. Psychol. 41, 49-100.

Mohr, P. N. C., Li, S. C., and Heekeren, H. R. (2010). Neuroeconomics and aging: neuromodulation of economic decision making in old age. Neurosci. Biobehav. Rev. 34, 678-688.

Moody, D. E. (2009). Can intelligence be increased by training on a task of working memory? Intelligence 37 , 327-328.

Nieuwenhuis, S., Ridderinkhof, K. R., De Jong, R., Kok, A., and Van Der Molen, M. W. (2000) Inhibitory inefficiency and failures of intention activation: age-related decline in the control of saccadic eye movements. Psychol. Aging 15, 635-647.

Noack, H., Lövden, M., Schmiedek, F., and Lindenberger, U. (2009). Cognitive plasticity in adulthood and old age: gauging the generality of cognitive intervention effects. Restor. Neurol. Neurosci. 27, 435-453.

O'brien, J. L., O'keefe, K. M., Laviolette, P. S., Deluca, A. N., Blacker, D., Dickerson, B. C., and Sperling, R. A. (2010). Longitudinal fMRI in elderly reveals loss of hippocampal activation with clinical decline. Neurology 74, 1969-1976.
O'sullivan, M., Jones, D. K., Summers, P. E., Morris, R. G., Williams, S. C., and Markus, H. S. (2001). Evidence for cortical "disconnection" as a mechanism of age-related cognitive decline. Neurology 57, 632-638.

Papp, K. V., Walsh, S. J., and Snyder, P. J. (2009). Immediate and delayed effects of cognitive interventions in healthy elderly: a review of current literature and future directions. Alzheimers Dement. 5, 50-60.

Persson, J., Nyberg, L., Lind, J., Larsson, A., Nilsson, L. G., Ingvar, M., and Buckner, R. L. (2006). Structurefunction correlates of cognitive decline in aging. Cereb. Cortex 16, 907.

Potter, G. G., Helms, M. J., Burke, J. R., Steffens, D. C., and Plassman, B. L. (2007). Job demands and dementia risk among male twin pairs. Alzheimers Dement. 3, 192-199.

Rabbitt, P., Lowe, C., and Shilling, V. (2001). Frontal tests and models for cognitive ageing. Eur. J. Cogn. Psychol. 13, 5-28.

Ranganath, C., and Rainer, G. (2003). Neural mechanisms for detecting and remembering novel events. Nat. Rev. Neurosci. 4, 193-202.

Raz, N., Lindenberger, U., Rodrigue, K. M., Kennedy, K. M., Head, D., Williamson, A., Dahle, C. Gerstorf, D., and Acker, J. D. (2005). Regional brain changes in aging healthy adults: general trends, individual differences and modifiers. Cereb. Cortex 15, 1676-1689.

Rebok, G. W., Carlson, M. C., and Langbaurn, J. B. S. (2007). Training and maintaining memory abilities in healthy older adults: traditional and novel approaches. J. Gerontol. B Psychol. Sci. Soc. Sci. 62, 53-61.

Reimers, S., Maylor, E. A., Stewart, N. and Chater, N. (2009). Associations between a one-shot delay discounting measure and age, income, education and real-world impulsive behavior. Pers. Individ. Dif. 47, 973-978.

Ridderinkhof, K. R., Forstmann, B. U., Wylie, S. A., Burle, B., and Van Den Wildenberg, W. P. M. (2011). Neurocognitive mechanisms of action control: resisting the call of the sirens. Wiley Interdiscip. Rev. Cogn. Sci. 2, 174-192.

Ridderinkhof, K. R., Van Den Wildenberg, W. P. M., Segalowitz, S. J., and Carter, C. S. (2004). Neurocognitive mechanisms of cognitive control: the role of prefrontal cortex in action selection, response inhibition, performance 
monitoring, and reward-based learning. Brain Cogn. 56, 129-140.

Roberts, B. A., Fuhrer, R., Marmot, M., and Richards, M. (2011). Does retirement influence cognitive performance? the whitehall II study. J. Epidemiol. Community Health 65, 958-963.

Salmon, E., Van Der Linden, M., Collette, F., Delfiore, G., Maquet, P., Degueldre, C., Luxen, A., and Franck, G. (1996). Regional brain activity during working memory tasks. Brain 119(Pt 5), 1617-1625.

Samanez-Larkin, G. R., Gibbs, S. E., Khanna, K., Nielsen, L., Carstensen, L. L., and Knutson, B. (2007). Anticipation of monetary gain but not loss in healthy older adults. Nat. Neurosci. 10, 787-791.

Samanez-Larkin, G. R., Wagner, A. D., and Knutson, B. (2011). Expected value information improves financial risk taking across the adult life span. Soc. Cogn. Affect. Neurosci. 6, 207.

Schliebs, R., and Arendt, T. (2010). The cholinergic system in aging and neuronal degeneration. Behav. Brain Res. 221, 555-563.

Schmiedek, F., Lövdén, M., and Lindenberger, U. (2010). Hundred days of cognitive training enhance broad cognitive abilities in adulthood: findings from the COGITO study. Front. Aging Neurosci. 2:27. doi: 10.3389/fnagi.2010.00027

Schmitt-Eliassen, J., Ferstl, R., Wiesner, C., Deuschl, G., and Witt, K. (2007). Feedback-based versus observational classification learning in healthy aging and Parkinson's disease. Brain Res. 1142, 178-188.

Schooler, C., Mulatu, M. S., and Oates, G. (1999). The continuing effects of substantively complex work on the intellectual functioning of older workers. Psychol. Aging 14, 483-506.

Schott, B. H., Niehaus, L., Wittmann, B. C., Schutze, H., Seidenbecher, C. I., Heinze, H. J., and Düzel, E. (2007). Ageing and early-stage Parkinson's disease affect separable neural mechanisms of mesolimbic reward processing. Brain 130, Verhaeghen, P., and Basak, C. (2005). 2412-2424.

Sharps, M. J., and Price-Sharps, J. L. (1996). Visual memory support: An effective mnemonic device for older adults. Gerontologist 36, 706-708.

Shipstead, Z., Redick, T. S., and Engle, R. W. (2010). Does working memory training generalize? Psychol. Belg. 50, 245-276.

Smith, G. E., Housen, P., Yaffe, K. Ruff, R., Kennison, R. F., Mahncke, H. W., and Zelinski, E. M. (2009). A cognitive training program based on principles of brain plasticity: results from the improvement in memory with plasticity-based adaptive cognitive training (IMPACT) study. J. Am. Geriatr. Soc. 57, 594-603.

Stigsdotter, A., and Bäckman, L. (1989). Multifactorial memory training with older adults - how to foster maintenance of improved performance. Gerontology 35, 260-267.

Strobach, T., Liepelt, R., Schubert, T. and Kiesel, A. (2011). Task switching: effects of practice on switch and mixing costs. Psychol. Res. 76, 74-83.

Thorell, L. B., Lindqvist, S., Nutley, S. B., Bohlin, G., and Klingberg, T. (2009). Training and transfer effects of executive functions in preschool children. Dev. Sci. 12, 106-113.

Treitz, F. H., Heyder, K., and Daum, I. (2007). Differential course of executive control changes during normal aging. Neuropsychol. Dev. Cogn. B Aging Neuropsychol. Cogn. 14, 370-393.

Van Der Linden, M., Collette, F., Salmon, E., Delfiore, G., Degueldre, C., Luxen, A., and Franck, G. (1999). The neural correlates of updating information in verbal working memory. Memory 7, 549-560.

Van Gerven, P. W., Meijer, W. A., Prickaerts, J. H., and Van Der Veen, F. M. (2008). Aging and focus switching in working memory: excluding the potential role of memory load. Exp. Aging Res. 34, 367-378.
Ageing and switching of the focus of attention in working memory: results from a modified N-back task. Q. J. Exp. Psychol. A 58, 134-154.

Verhaeghen, P., Steitz, D. W., Sliwinski, M. J., and Cerella, J. (2003). Aging and dual-task performance: a meta-analysis. Psychol. Aging 18, 443-460.

Volkow, N. D., Gur, R. C., Wang, G. J., Fowler, J. S., Moberg, P. J., Ding, Y. S., Hitzemann, R., Smith, G., and Logan, J. (1998). Association between decline in brain dopamine activity with age and cognitive and motor impairment in healthy individuals. Am. J. Psychiatry 155, 344-349.

Voss, M. W., Prakash, R. S., Erickson, K. I., Basak, C., Chaddock, L., Kim, J. S., Alves, H., Heo, S., Szabo, A. N., and White, S. M. (2010) Plasticity of brain networks in a randomized intervention trial of exercise training in older adults. Front. Aging Neurosci. 2:32. doi: 10.3389/fnagi.2010.00032

Waiter, G. D., Fox, H. C., Murray, A. D., Starr, J. M., Staff, R. T., Bourne, V. J., Whalley, L. J., and Deary, I. J. (2008). Is retaining the youthful functional anatomy underlying speed of information processing a signature of successful cognitive ageing? An eventrelated fMRI study of inspection time performance. Neuroimage 41, 581-595.

Wang, G. J., Volkow, N. D., Logan, J. Fowler, J. S., Schlyer, D., Macgregor, R. R., Hitzemann, R. J., Gur, R. C., and Wolf, A. P. (1995). Evaluation of age-related changes in serotonin 5-HT2 and dopamine D2 receptor availability in healthy human subjects. Life Sci. 56, PL249-PL253.

Wang, Y., Chan, G. L., Holden, J. E. Dobko, T., Mak, E., Schulzer, M., Huser, J. M., Snow, B. J., Ruth, T. J., Calne, D. B., and Stoessl, A. J. (1998). Age-dependent decline of dopamine D1 receptors in human brain: a PET study. Synapse 30, 56-61.
Wecker, N. S., Kramer, J. H., Hallam, B. J., and Delis, D. C. (2005). Mental flexibility: age effects on switching. Neuropsychology 19, 345-352.

Williams, B. R., Ponesse, J. S., Schachar, R. J., Logan, G. D., and Tannock, R. (1999). Development of inhibitory control across the life span. Dev. Psychol. 35, 205.

Winocur, G. (1998). Environmental influences on cognitive decline in aged rats. Neurobiol. Aging 19, 589-597.

Yaffe, K., Fiocco, A. J., Lindquist, K., Vittinghoff, E., Simonsick, E. M., Newman, A. B., Satterfield, S., Rosano, C., Rubin, S. M., Ayonayon, H. N., and Harris, T. B. (2009). Predictors of maintaining cognitive function in older adults: the health ABC study. Neurology 72, 2029-2035

Yates, F. (1966). The Art of Memory. London: Routledge and Kegan Paul Ltd.

Zelinski, E. M. (2009). Far transfer in cognitive training of older adults. Restor. Neurol. Neurosci. 27, 455-471.

Conflict of Interest Statement: The authors declare that the research was conducted in the absence of any commercial or financial relationships that could be construed as a potential conflict of interest.

Received: 01 February 2012; accepted: 01 June 2012; published online: 21 June 2012.

Citation: Buitenweg JIV, Murre JMJ and Ridderinkhof KR (2012) Brain training in progress: a review of trainability in healthy seniors. Front. Hum. Neurosci. 6:183. doi: 10.3389/fnhum.2012.00183 Copyright (c) 2012 Buitenweg, Murre and Ridderinkhof. This is an openaccess article distributed under the terms of the Creative Commons Attribution Non Commercial License, which permits non-commercial use, distribution, and reproduction in other forums, provided the original authors and source are credited. 